\title{
Short wave diathermy for small spontaneous pneumothorax
}

\author{
Yingmin Ma, Jihua Li, Youning Liu
}

\begin{abstract}
Background - The treatment of small spontaneous pneumothorax can involve observation, tube thoracostomy, and surgery. This study evaluated the use of short wave diathermy as a method of accelerating the resolution of small pneumothoraces.

Methods - Twenty two patients with pneumothoraces of less than $30 \%$ by volume were randomly allocated to receive short wave diathermy for 25 minutes each day $(n=11)$ or observation with bed rest $(n=$ 11). Chest radiographs were taken until the pneumothoraces resolved.

Results - There were no significant differences in the clinical characteristics between the two groups of patients. However, the mean (SD) rate of absorption was significantly higher with short wave diathermy than with observation (3.44 $(0.94) \%$ versus $1.57(0.53) \%$, difference $=$ $1.87,95 \%$ confidence interval (CI) 1.19 to $2.55, \mathrm{p}<0.001)$. The time to complete reexpansion was shorter with short wave diathermy than with observation (6.86 (3.51) days versus 11.64 (3.61) days, difference = -4.78 days, $95 \%$ CI -7.95 to -1.61 , $p<0.005)$. No evidence of damage resulting from short wave diathermy was found. Conclusions - Although further study is necessary, these results indicate that short wave diathermy may be an alternative treatment for patients with small spontaneous pneumothoraces.

(Thorax 1997;52:561-562)
\end{abstract}

Keywords: spontaneous pneumothorax, short wave diathermy, therapeutics.

Department of Respiratory Medicine Y Ma

Y Liu

Department of Physiotherapy

$\mathrm{J} \mathrm{Li}$

PLA General Hospital, 28 Fuxing Road, Beijing, China 100853

Correspondence to: Dr Y Ma.

Received 25 July 1996 Returned to authors 2 October 1996

Revised version received

24 December 1996

Accepted for publication

2 January 1997 or conservative management are probably the most common treatment for patients with residual pneumothorax and asymptomatic patients with less than $20 \%$ pneumothoraces by volume, relying on the pleural surface to reabsorb the intrapleural air.

It is generally agreed that short wave diathermy increases the blood flow of local tissue. Therapeutic benefits of short wave diathermy are apparently connected with the relationship thought to exist between tissue temperature and blood flow. ${ }^{5}$ We hypothesised that the change in temperature of the pleural cavity and the increased blood flow of the pleural capillary caused by short wave diathermy might increase the rate of absorption of gas from the pleural cavity. The present study was designed to evaluate the efficacy of short wave diathermy in the treatment of small spontaneous pneumothorax.

\section{Methods}

Asymptomatic patients with less than 30\% pneumothoraces by volume and patients with a residual pneumothorax after thoracocentesis or thoracic drainage were included in the study. Those with a tension pneumothorax and pregnant women were excluded. Twenty two patients diagnosed with spontaneous pneumothorax and treated in our hospital who had refused placement of chest tubes or other surgical procedures were randomly divided into two groups of 11 patients. Group 1 (nine men) were treated with short wave diathermy and group 2 (10 men) were treated with bed rest and observation. The size of the pneumothorax was estimated according to the method of Light $^{6}$ by measuring the mean diameter of the lung and the thorax, cubing these diameters, and finding the ratios. The study was approved by the subcommittee on human study of the PLA General Hospital.

Short wave diathermy (LDT.CD31, Beijing Medical Apparatus Co., China) was applied to a field of the upper thorax on the affected side with an intensity that caused a warmth that was just perceptible. It was applied once a day for 25 minutes with the subject lying supine on a bed. Chest radiographs were taken in all patients on full inspiration every two days. and were read by two radiologists who were blinded to the treatment allocated. The mean rate of absorption was estimated by the ratio of the size 
Table 1 Clinical characteristics of patients

\begin{tabular}{llll}
\hline Patient no. & Age (years) & Size of pneumothorax (\%) & Underlying lung disease \\
\hline Group 1 & & & \\
1 & 17 & 15 & Asthma \\
2 & 23 & 10 & Asthma \\
3 & 36 & 20 & None \\
4 & 28 & 25 & None \\
5 & 28 & 15 & Cone \\
6 & 59 & 10 & None \\
7 & 20 & 30 & None \\
8 & 20 & 45 & None \\
9 & 32 & 30 & Chronic bronchitis \\
10 & 63 & 20 & None \\
11 & 28 & 20 & \\
Group 2 & & 20 & Asthma \\
1 & 17 & 20 & None \\
2 & 18 & 10 & None \\
3 & 30 & 10 & None \\
4 & 20 & 20 & Chronic bronchitis \\
5 & 20 & 30 & None \\
6 & 51 & 10 & None \\
7 & 24 & 15 & None \\
8 & 26 & 20 & None \\
9 & 20 & & \\
10 & 33 & &
\end{tabular}

Table 2 Mean (SD) data of patients receiving short wave diathermy (group 1) and those treated with observation and bed rest (group 2)

\begin{tabular}{llll}
\hline & Group 1 & Group 2 & p value \\
\hline Age (years) & $32.18(15.32)$ & $29.36(12.94)$ & NS \\
Size of pneumothorax (\%) & $22.08(9.88)$ & $17.92(5.82)$ & NS \\
Time to complete re-expansion (days) & $6.86(3.51)$ & $11.64(3.61)$ & $<0.005$ \\
Rate of absorption (\%) & $3.44(0.94)$ & $1.57(0.53)$ & $<0.001$ \\
\hline
\end{tabular}

of the pneumothorax to the time to complete reexpansion as the following equation.

Mean rate of absorption (\%/day) $=$

$$
\frac{\text { Size of pneumothorax (\%) }}{\text { Time to complete re-expansion (days) }}
$$

The results are presented as mean (SD) and comparisons between quantitative values were made with the Student's $t$ test.

\section{Results}

Table 1 shows the clinical characteristics of both groups of patients. The time to re-expansion for the patients in group 1 varied from 3 to 12.5 days and for those in group 2 from 8 to 17 days. The rate of absorption in patients in group 2 ranged from $1 \%$ to $2.5 \%$ compared with $2 \%$ to $5 \%$ in group 1 .

Table 2 shows a comparison of the quantitative values between the two groups. There were no significant differences in the clinical data between the two groups, but the time to re-expansion in the patients who received short wave diathermy was shorter than in those who underwent conservative management $(\mathrm{p}<0.005)$ and the mean rate of absorption in the treatment group was increased. The time to re-expansion was reduced by 4.78 days ( $95 \%$ CI 1.61 to 7.95 ) and the rate of absorption was increased by $1.87 \%$ (95\% CI 1.19 to 2.55 ) in those receiving short wave diathermy compared with observation and bed rest.

\section{Discussion}

To our knowledge this is the first report describing the effect of short wave diathermy in the treatment of spontaneous pneumothorax. It is generally accepted that short wave diathermy, which provides the deepest heat available because of the high frequency waves of electromagnetic radiation, plays an important part in the treatment of most musculoskeletal disorders. ${ }^{7}$ Sekins ${ }^{5}$ and Lehmann et al ${ }^{8}$ showed that increased blood flow resulted in an increased rate of resolution of muscle haematoma by enhancing the vascular washout of the red blood cell breakdown products. This suggests that increased blood flow of the pleural capillary might enhance the absorption of intrapleural air. On the other hand, the heat supplied to the pleural cavity results in an increase in the temperature of the intrapleural air. According to the theory of molecular physics the pressure of a gas is proportional to its temperature if the volume of the gas is constant. During treatment with short wave diathermy the volume of the pleural cavity remains nearly constant so the increased pressure of the intrapleural air might increase the pressure difference between it and the air in a pleural capillary which might also accelerate the rate of absorption. In the present series treatment with short wave diathermy resulted in a twofold increase in the mean rate of absorption and shortened the course of treatment.

Although supplemental oxygen at a high concentration may facilitate resolution of the pneumothorax by increasing the nitrogen gradient,${ }^{9}$ the toxic effect of a high concentration of oxygen on the lungs and the discomfort of wearing a face mask continuously for more than 10 hours restricts its use.

Our results suggest that simple short wave diathermy is an effective alternative treatment for small spontaneous pneumothoraces which merits further study.

1 Seremetis MG. The management of spontaneous pneumothorax. Chest 1970;57:65-8.

2 Abolnik IZ, Lossos IS, Gillis D, et al. Primary spontaneous pneumothorax in men. Am f Med Sci 1993;305:297-303.

3 British Thoracic Society Research Committee. Comparison of simple aspiration with intercostal drainage in the management of spontaneous pneumothorax. Thorax 1993;48. 430-1.

4 Andrivet $\mathrm{P}$, Djedaini $\mathrm{K}$, Teboul JL, et al. Spontaneous pneumothorax: comparison of thoracic drainage vs immediate or delayed needle aspiration. Chest 1995;108:33540 .

5 Sekins KM, Lehmann JF, Esselman PC, et al. Local muscle blood flow and temperature responses to $915 \mathrm{MHz}$ diathermy as simultaneously and numerically predicted. Arch Phys Med Rehabil 1984;65:1-7.

6 Light RW. Management of spontaneous pneumothorax. Am Rev Respir Dis 1993;148:245-8.

7 Jan MH, Lai JS. The effect of physiotherapy on osteoarthritic knees of females. F Formosan Med Assoc 1991;90:1008-11

8 Lehmann JF, Doundore DE, Esselman PC, et al. Microwave diathermy: effects on experimental muscle hematoma resolution. Arch Phys Med Rehabil 1983;64:127-30.

9 Northfield TC. Oxygen therapy for spontaneous pneumothorax. BMF 1971;4:86-8. 